\title{
Pancreatic biopsies in type 1 diabetes: revisiting the myth of Pandora's box
}

\author{
Mark A. Atkinson
}

Received: 13 December 2013 / Accepted: 16 December 2013 /Published online: 19 January 2014

(C) Springer-Verlag Berlin Heidelberg 2014

\begin{abstract}
Over a century ago, inquisitive physicians made remarkable discoveries regarding pancreatic pathology in individuals with diabetes, including those who were likely afflicted with the type 1 (autoimmune) form of the disease. Those studies of post-mortem tissues noted unique anatomical changes in islet architecture as well as the presence of unusual cellular infiltrates. In the time since, investigations of pancreatic pathology have, with near uniformity, been restricted to analysis of organs obtained post-mortem. While clearly beneficial for addressing questions of the disorder's pathogenesis, concern exists regarding potential artefacts that might occur through analysis of tissues that have been recovered hours, often many hours, following death. Beyond this, studies of tissues obtained long after the diagnosis of type 1 diabetes may not disclose important physiological events occurring at onset or even earlier in the natural history of disease, before symptomatic hyperglycaemia. To this end, Krogvold and colleagues (in this issue of Diabetologia, doi:10.1007/s00125013-3155-y) undertook a potentially high-reward strategy involving pancreatic biopsy in living adults with recentonset type 1 diabetes. Procedures were performed under informed consent, undertaken based on recent improvements in laparoscopic techniques, and carried out by individuals with considerable surgical experience. These efforts were terminated for ethical reasons following the occurrence of serious complications (including post-operative bleeding and pancreatic leakage). The experience lends itself to analogy with the Greek myth of Pandora's box where curiosity, in terms of a desire to see what resided inside a closed container, unleashed a series of ills on humans once the container was opened. In considering the moral of that myth, one must question whether
\end{abstract}

M. A. Atkinson $(\bowtie)$

Department of Pathology, College of Medicine, University of Florida, 1600 SW Archer Road, Gainesville, FL 32610-0275, USA

e-mail: atkinson@ufl.edu the secrets of the pancreas in those living with type 1 diabetes should, for now, remain a mystery as the process of manipulating that organ for the purpose of curiosity does not occur without harm.

Keywords Biopsy · Immunology · Inflammation · Innate immunity $\cdot$ Pancreas $\cdot$ Pathology $\cdot$ Surgical complications . Type 1 diabetes

\section{Abbreviation \\ DiViD Diabetes Virus Detection Study}

\section{The myth of Pandora's box}

The myth of Pandora's box — which, for full disclosure, may more accurately be translated as 'jar'-remains one of the most oft described accounts in Greek mythology [1]. Pandora, according to the myth, was not only the first woman on Earth but also one given a variety of gifts by her god creators. Those gifts resided in a box, given to her by the gods, with the strict order never to open the box, ever. While Pandora attempted to tame her curiosity, in the end she succumbed to temptation and, after stealing keys from her husband Epimetheus, opened the box. The result was a release of all the illnesses and hardships hidden inside by the gods. Being scared, Pandora rapidly tried to close the box and, in doing so, closed hope inside.

\section{Why begin a commentary with a story thousands of years old?}

Insights from the story of Pandora's box have been fascinating individuals for generations, catching the 
imagination of storytellers, artists, psychologists and, as in this situation, a modern day scientific commentator. My reason for sharing this legend resides in its remarkable analogy to recent attempts seeking to answer the question: 'What resides in the pancreas of those living with type 1 diabetes?'.

To be clear, previous studies of pancreatic pathology have already shed some light on the pathogenic nature of the disease and its heterogeneity. A broad body of evidence supports the notion that type 1 diabetes is a disorder resulting from an autoimmune destruction of pancreatic beta cells [2]. This concept finds much of its basis in decades-old observations involving post-mortem tissues that portend, at the symptomatic onset of type 1 diabetes, the presence of a chronic inflammatory infiltrate, often termed 'insulitis', in the majority of subjects [3]. Immunophenotyping efforts utilising these tissues suggest the infiltrate consists predominantly of $\mathrm{T}$ lymphocytes, with lesser numbers of B lymphocytes and macrophages [4]. Diminished insulin staining, hyper-expression of class I MHC on all endocrine cells in insulin-containing islets, and the presence of pseudoatrophic islets (i.e. islets devoid of insulin-producing cells) are also noteworthy occurrences with this lesion $[5,6]$. Studies of pancreatic pathology also highlight another greatly underappreciated yet important point: that type 1 diabetes is likely to be a collection of different diseases united by a common outcome (i.e. destruction of beta cells and increased blood glucose levels) but differing in their pathogenesis [7].

While clearly informative, studies of post-mortem pancreases from recent-onset type 1 diabetic patients are, however, limited by matters of both form and fashion. Form, in that we now appreciate that type 1 diabetes is a disorder where the immune-mediated destruction of beta cells appears to occur over a period of many months to years, perhaps even in a relapsingremitting fashion [2]. The inability to access pancreatic specimens prior to symptomatic onset is likely to leave features important to our understanding of the disease unknown. With respect to fashion, the use of autopsybased specimens has been criticised for their potential to mask important information or instil artefacts that incorrectly guide interpretation, both leading to intellectual inaccuracies. Indeed, post-mortem tissues are often subject to varying degrees of autolysis. Examples of previously described pancreatic features that have been criticised for this reason include the notions of beta cell apoptosis, beta cell replication, and the expression of Fas by beta cells, amongst others. So how would one move beyond this situation? These issues of form and fashion could, in theory, be overcome by the introduction of pancreatic biopsy in individuals with type 1 diabetes, as well as in those at increased risk for the disorder (i.e. people possessing antiislet cell autoantibodies).

\section{Why open the box?}

Pancreatic biopsies of living individuals have the potential to provide vital information on numerous questions relevant to the pathogenesis of type 1 diabetes including, but not limited to, those noted in the text box. Considered together, this information would not only allow for improved answers to the question of why type 1 diabetes occurs, but in addition, it has the potential to undercover new therapeutic avenues to prevent and/or reverse the disease.

Garnering information that would direct the design of improved therapeutics is an exceptionally important requirement as, to date, such efforts have largely relied on information derived from studies of peripheral blood, animal models of the disease (e.g. NOD mice), or what ultimately amounts to an educated guess based on matching a particular agent to a presumed pathogenic feature of the disease. While progress has been made, an intervention capable of preventing or durably reversing type 1 diabetes has, to date, proven elusive. Hence, for a number of reasons, the idea of opening the box (i.e. the pancreas) is a very tempting one.

\section{Knowledge voids that might be influenced by the availability of biopsy material from patients with recent-onset type 1 diabetes}

- The role of viruses in the disorder's pathogenesis

- Improved characterisation of the cellular phenotype comprising the insulitis lesion

- Better evaluation of islet morphology and composition

- Identifying evidence for beta cell regeneration

- Determining potential mechanisms of beta cell death

- Establishing the degree of beta cell loss at a given period in the natural history of the disease

\section{The outcomes of opening the box}

Armed with such curiosity and noble in purpose, Krogvold and colleagues attempted a small series of pancreatic biopsies or, more accurately, tail resections, in individuals with recentonset disease [8]. The effort was carried out as part of the Diabetes Virus Detection (DiViD) project, a collective of experts representing a wide swathe of medical disciplines, including gastroenterological surgery. All appropriate ethics and performance-based approvals were obtained, including 
that of a governmental regional ethics committee, and informed consent was obtained from participating individuals. Beyond tail resection, duodenal biopsies were also undertaken to gain insights into intestinal inflammation and a potential gateway for viruses. In terms of actual performance, six participants (all adults) with type 1 diabetes of short duration (3 to 9 weeks post diagnosis) underwent biopsy.

While the procedure was relatively uncomplicated for three participants, this was not the case for the remainder. Indeed, adverse events in the remaining individuals included extensive post-operative bleeding, pancreatic drainage, splenic tear, pain and fever. These events resulted in the need for an extensive, unplanned duration of hospital stay. While the patients all recovered fully, the DiViD investigators themselves determined it wise to end the study and cease further recruitment.

At this point, a number of obvious questions arise, perhaps the most predominant one being: 'Was this effort worth it?' Unfortunately, the answer cannot be provided until the data emanating from studies using the tissues are available. Hence, time will allow for better judgement of that issue. Beyond this, additional questions include: 'Why did complications arise in some patients and not in others?'; 'Should the study have been stopped?'; and perhaps for those most concerned about safety: 'Should the study have been undertaken in the first place?' An additional curious aspect of the Krogvold et al study [8] involves comparisons of its safety outcomes with those of a team of Japanese investigators some 15 years ago [9]. In the earlier study, complication rates were minimal in comparison to this modern effort, perhaps due to the Japanese study using a more traditional punch biopsy based approach, gaining small amounts of tissue with very few islets. That said, the potential research benefits, in terms of understanding the unknowns in type 1 diabetes (see the text box), could be far greater with the superior quantities of material that can be obtained with partial resection. To that end, one might also question whether lessons learned from this effort might lead to additional surgical improvements that would, in fact, allow for such procedures to be performed in a safe fashion.

\section{Conclusions}

So, what conclusions can we glean from opening this Pandora's box in type 1 diabetes? First, the authors of this work should be commended for their honesty and transparency in sharing their experiences. The message is an important one that the community of diabetes researchers must hear. Second, one must question whether their attempts to perform these biopsies were worth the complications that resulted. This is an important question and one that unfortunately, at present, cannot be fully answered due to the lack of scientific information from the biopsies obtained; there is great anticipation regarding these results. Next, attempts to understand the inner workings of the pancreas in type 1 diabetic patients, living or deceased, should not stop with this setback. Indeed, if anything, this outcome should bolster alternative approaches to evaluate the inner workings of the pancreas, including and especially pancreatic imaging via NMR or PET [10]. These efforts also provide support, albeit indirect, for initiatives designed to collect whole pancreases from organ donors, such as PanFin and the Network for Pancreatic Organ Donors with Diabetes (nPOD) (www.jdrfnpod.org) [11, 12]. These efforts not only evaluate cases with type 1 diabetes but, in addition, presumed 'prediabetic' pancreases obtained from non-diabetic individuals who possess anti-islet cell autoantibodies. Finally, one must consider the overall question of whether pancreatic biopsies should be performed in type 1 diabetic patients for research purposes. Based on the experience of Krogvold and colleagues [8], the most appropriate response to this would clearly appear to be 'no'. However, only when the data obtained from these studies come in, and we can observe where this information guides the field regarding the prevention or cure of the disease, will we be in a position to address whether what, in the end, turned out to be a high-risk procedure was warranted.

Acknowledgements This work was supported, in part, by funding obtained from The National Institutes of Health, The JDRF, The Brehm Coalition for Type 1 Diabetes Research, and the Jeffrey Keene Family Professorship.

Duality of interest The author declares that there is no duality of interest associated with this manuscript.

Contribution statement The author notes that he is the sole composer of the Commentary and the opinions expressed reflect his own beliefs.

\section{References}

1. Verdenius WJ (1985) A commentary on Hesiod: works and days, vv 1-382. EJ Brill, Leiden

2. Atkinson M, Eisenbarth GS, Michels A (2014) Type 1 diabetes. Lancet 383:69-82

3. Gepts W (1965) Pathologic anatomy of the pancreas in juvenile diabetes mellitus. Diabetes 14:619-633

4. Richardson SJ, Willcox A, Bone AJ, Morgan NG, Foulis AK (2011) Immunopathology of the human pancreas in type-I diabetes. Semin Immunopathol 33:9-21

5. Coppieters KT, Dotta F, Amirian N et al (2012) Demonstration of islet-autoreactive CD8 T cells in insulitic lesions from recent onset and long-term type 1 diabetes patients. J Exp Med 209:51-60

6. Gianani R, Campbell-Thompson M, Sarkar SA et al (2010) Dimorphic histopathology of long standing childhood onset diabetes. Diabetologia 53:1811-1822

7. Atkinson MA, Gianani R (2009) The pancreas in human type 1 diabetes - providing new answers to age old questions. Curr Opin Diabetes Endocrinol Obes 16:279-285

8. Krogvold L, Edwin B, Buanes T et al (2013) Pancreatic biopsy by minimal tail resection in live adult patients at the onset of type 1 diabetes: experiences from the DiViD study. Diabetologia. doi:10.1007/s00125-013-3155-y 
9. Imagawa A, Hanafusa T, Tamura S et al (2001) Pancreatic biopsy as a procedure for detecting in situ autoimmune phenomena in type 1 diabetes: close correlation between serological markers and histological evidence of cellular autoimmunity. Diabetes 50:1269-1273

10. Di Gialleonardo V, de Vries EF, Di Girolamo M, Quintero AM, Dierckx RA, Signore A (2012) Imaging of $\beta$-cell mass and insulitis in insulin-dependent (type 1) diabetes mellitus. Endocr Rev 33:892-919
11. Tauriainen S, Salmela K, Rantala I, Knip M, Hyöty H (2010) Collecting high-quality pancreatic tissue for experimental study from organ donors with signs of $\beta$-cell autoimmunity. Diabetes Metab Res Rev 26:585-592

12. Campbell-Thompson M, Albanese-O'Neill A, Staeva T et al (2012) The network for pancreatic organ donors with diabetes (nPOD): tissue biobanking for the purpose of understanding the causes of and cures for type 1 diabetes. Diabetes Metab Res Rev 28(7):608-617 